UWThPh-2002-29

\title{
Global analysis of neutrino oscillation data in four-neutrino schemes
}

\author{
M. Maltoni ${ }^{\mathrm{a}}$, T. Schwetz ${ }^{\mathrm{b} *}$, M. A. Tórtola ${ }^{\mathrm{a}}$ and J. W. F. Valle ${ }^{\mathrm{a}}$ \\ ${ }^{a}$ Instituto de Física Corpuscular - C.S.I.C./Universitat de València \\ Edificio Institutos de Paterna, Apt 22085, E-46071 Valencia, Spain \\ bInstitut für Theoretische Physik, Universität Wien \\ Boltzmanngasse 5, A-1090 Wien, Austria
}

We present an analysis of the global neutrino oscillation data in terms of four-neutrino mass schemes. We find that the strong preference of oscillations into active neutrinos implied by the latest solar as well as atmospheric neutrino data allows to rule out $(2+2)$ mass schemes, whereas $(3+1)$ schemes are strongly disfavoured by shortbaseline experiments. Our analysis shows that four-neutrino oscillations do not provide a satisfactory description of the global neutrino oscillation data including the LSND result.

\section{INTRODUCTION}

The neutrino oscillation interpretations of the solar [1,2] and atmospheric [3, [4] neutrino data and the LSND experiment [5] require three neutrino mass-squared differences of different orders of magnitude. Since it is not possible to obtain this within the Standard Model framework of three active neutrinos it has been proposed to introduce a light sterile neutrino [6] to reconcile all the experimental hints for neutrino oscillations. Here we present an analysis of the global neutrino oscillation data in terms of fourneutrino mass schemes, including data from solar and atmospheric neutrino experiments, the LSND experiment, as well as data from short-baseline (SBL) experiments [7,8,9] and long-baseline reactor experiments 10 reporting no evidence for oscillations. This analysis updates the work presented at the meeting and is based on the data of summer 2002. We find that for all possible types of four-neutrino schemes different sub-sets of the data are in serious disagreement and hence, four-

\footnotetext{
*Talk given by T.S. at the XXX International Meeting on Fundamental Physics, Jaca, Spain, 28 Jan - 1 Feb 2002. This work has been supported by the ESF network 86 , the Marie Curie Training Grant HPMT-2000-00124 and the DOC fellowship of the Austrian Academy of Science.
}

neutrino oscillations do not provide a satisfactory description of the global oscillation data including LSND. The details of our calculations can be found in Refs. 11, 12, 13 .

Four-neutrino mass schemes are usually divided into the two classes $(3+1)$ and $(2+2)$, as illustrated in Fig. 11. We note that $(3+1)$ mass spectra include the three-active neutrino scenario as limiting case. In this case solar and atmospheric neutrino oscillations are explained by active neutrino oscillations, with mass-squared differences $\Delta m_{\mathrm{SOL}}^{2}$ and $\Delta m_{\mathrm{ATM}}^{2}$, and the fourth neutrino state gets completely decoupled. We will refer to such limiting scenario as $(3+0)$. In contrast, the $(2+2)$ spectrum is intrinsically different, as there must be a significant contribution of the sterile neutrino either in solar or in atmospheric neutrino oscillations or in both.

\section{NOTATIONS AND APPROXIMA- TIONS}

Neglecting CP violation, in general neutrino oscillations in four-neutrino schemes are described by 9 parameters: 3 mass-squared differences and 6 mixing angles in the unitary lepton mixing matrix. Here we use a parameterization introduced 

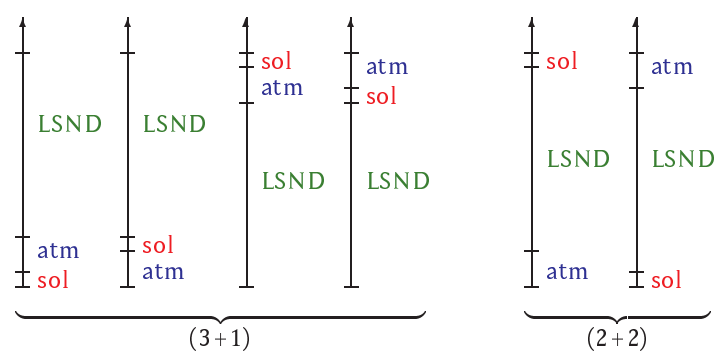

Figure 1. The six types of four-neutrino mass spectra, divided into the classes $(3+1)$ and $(2+2)$.

in Ref. [11], which is based on physically relevant quantities: the 6 parameters $\Delta m_{\mathrm{SOL}}^{2}, \theta_{\mathrm{SOL}}$, $\Delta m_{\mathrm{ATM}}^{2}, \theta_{\mathrm{ATM}}, \Delta m_{\mathrm{LSND}}^{2}, \theta_{\mathrm{LSND}}$ are similar to the two-neutrino mass-squared differences and mixing angles and are directly related to the oscillations in solar, atmospheric and the LSND experiments. For the remaining 3 parameters we use $\eta_{s}, \eta_{e}$ and $d_{\mu}$. Here, $\eta_{s}\left(\eta_{e}\right)$ is the fraction of $\nu_{s}\left(\nu_{e}\right)$ participating in solar oscillations, and $\left(1-d_{\mu}\right)$ is the fraction of $\nu_{\mu}$ participating in oscillations with $\Delta m_{\mathrm{ATM}}^{2}$ (for exact definitions see Ref. [11). For the analysis we adopt the following approximations:

- We make use of the hierarchy

$$
\Delta m_{\mathrm{SOL}}^{2} \ll \Delta m_{\mathrm{ATM}}^{2} \ll \Delta m_{\mathrm{LSND}}^{2} .
$$

This means that for each data set we consider only one mass-squared difference, the other two are set either to zero or to infinity.

- In the analyses of solar and atmospheric data (but not for SBL data) we set $\eta_{e}=1$, which is justified because of strong constraints from reactor experiments [9, 10].

Due to these approximations the parameter structure of the four-neutrino analysis gets rather simple. The parameter dependence of the different data sets solar, atmospheric and SBL is illustrated in Fig. 2. We see that only $\eta_{s}$ links solar and atmospheric data and $d_{\mu}$ links atmospheric and SBL data. All the other parameters are "private" to one data set.

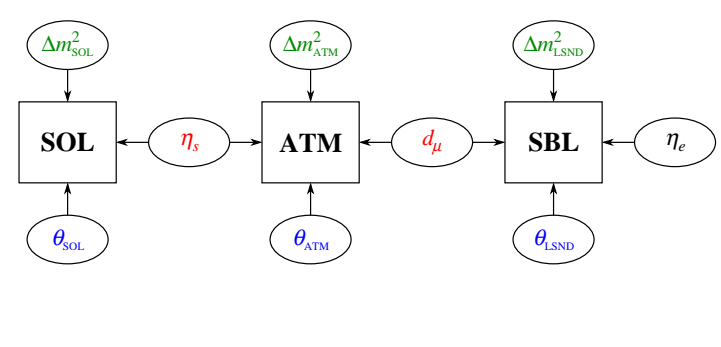

Figure 2. Parameter dependence of the different data sets in our parameterization.

\section{3. (2+2): RULED OUT BY SOLAR AND ATMOSPHERIC DATA}

The strong preference of oscillations into active neutrinos in solar and atmospheric oscillations leads to a direct conflict in $(2+2)$ oscillation schemes. We will now show that thanks to the new SNO solar neutrino data [2] and the improved SK statistic on atmospheric neutrinos [3] the tension in the data has become so strong that $(2+2)$ oscillation schemes are essentially ruled out Indeed, latest solar neutrino data lead to the bound $\eta_{s} \leq 0.45$ at $99 \% \mathrm{CL}$, where $\eta_{s}$ is the parameter describing the fraction of the sterile neutrino participating in solar neutrino oscillations. In contrast, in $(2+2)$ schemes atmospheric data imply $\eta_{s} \geq 0.65$ at $99 \% \mathrm{CL}$, in clear disagreement with the bound from solar data.

In Fig. 3 we show the $\chi^{2}$ for solar data and for atmospheric combined with SBL data as a function of $\eta_{s}$. Furthermore, we show the $\chi^{2}$ of the global data defined by

$\bar{\chi}^{2}\left(\eta_{s}\right) \equiv \Delta \chi_{\mathrm{SOL}}^{2}\left(\eta_{s}\right)+\Delta \chi_{\mathrm{ATM}+\mathrm{SBL}}^{2}\left(\eta_{s}\right)$.

From the figure we find that only if we take both data sets at the $99.95 \%$ CL a value of $\eta_{s}$ exists, which is contained in the allowed regions of both sets. This follows from the $\chi^{2}$-value $\chi_{\mathrm{PC}}^{2}=12.2$ shown in the figure. In Ref. 12 we have proposed a statistical method to evaluate the disagreement of different data sets in global analyses. The $p a-$ rameter goodness of fit (PG) makes use of the $\bar{\chi}^{2}$

${ }^{2}$ Details of our analyses of the solar_and atmospheric neutrino data can be found in Ref. [13. For an earlier four-neutrino analysis of solar and atmospheric data see Ref. 14. 


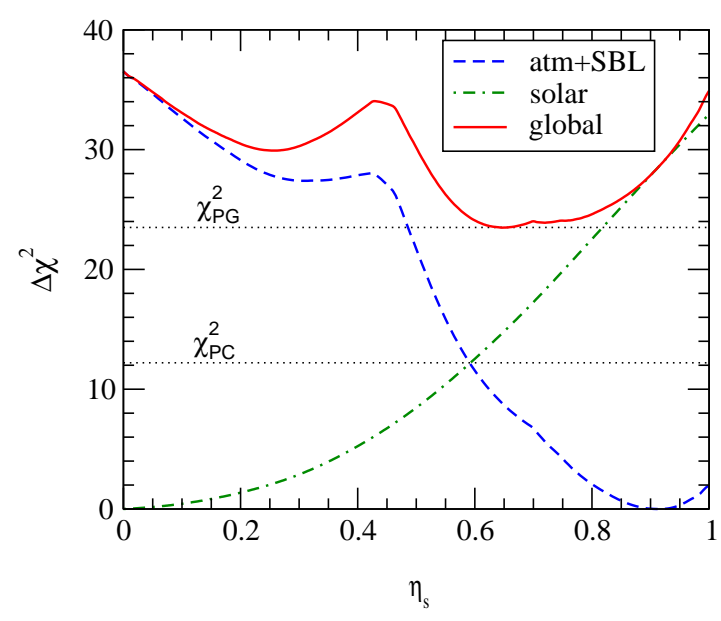

Figure 3. $\Delta \chi_{\mathrm{SOL}}^{2}, \Delta \chi_{\mathrm{ATM}+\mathrm{SBL}}^{2}$ and $\bar{\chi}_{\text {global }}^{2}$ as a function of $\eta_{s}$ in $(2+2)$ oscillation schemes.

defined in Eq. (2). This criterion evaluates the GOF of the combination of data sets, without being diluted by a large number of data points, as it happens for the usual GOF criterion (for details see Ref. [12]). We find $\chi_{\mathrm{PG}}^{2} \equiv \bar{\chi}_{\min }^{2}=23.5$, leading to the marginal PG of $1.3 \times 10^{-6}$. We conclude that $(2+2)$ oscillation schemes are highly disfavoured by the disagreement between the latest solar and atmospheric neutrino data. This is a very robust result, independent of whether LSND is confirmed or disproved.

\section{4. (3+1): STRONGLY DISFAVOURED BY SBL DATA}

It is known for a long time [15] that $(3+1)$ mass schemes are disfavoured by the comparison of SBL disappearance data [8,9] with the LSND result. In Ref. 16] we have calculated an upper bound on the LSND oscillation amplitude $\sin ^{2} 2 \theta_{\mathrm{LSND}}$ resulting from SBL and atmospheric neutrino data. From Fig. A we see that this bound is incompatible with the signal observed in LSND at the $95 \%$ CL. Only marginal overlap regions exist between the bound and LSND if both are taken at $99 \%$ CL. An analysis in terms of the parameter goodness of fit [12] shows that for

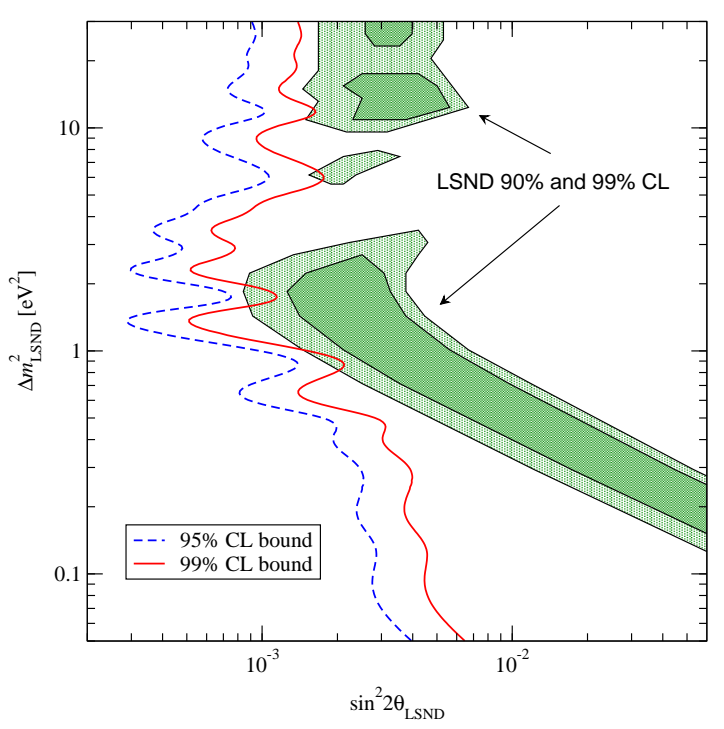

Figure 4. Upper bound on $\sin ^{2} 2 \theta_{\mathrm{LSND}}$ from SBL and atmospheric neutrino data in $(3+1)$ schemes [16] compared to the LSND allowed region [5].

most values of $\Delta m_{\mathrm{LSND}}^{2}$ SBL and atmospheric data are compatible with LSND only at more than $3 \sigma$, with one exception around $\Delta m_{\mathrm{LSND}}^{2} \sim 6 \mathrm{eV}^{2}$, where the PG reaches $1 \%$. These results show that $(3+1)$ schemes are strongly disfavoured by SBL disappearance data.

\section{COMPARING $(3+1),(2+2)$ AND $(3+0)$ HYPOTHESES}

With the methods developed in Ref. 11] we are able to perform a global fit to the oscillation data in the four-neutrino framework. This approach allows to statistically compare the different hypotheses. Let us first evaluate the GOF of $(3+1)$ and $(2+2)$ spectra with the help of the PG method described in Ref. [12]. We divide the global oscillation data in the 4 data sets SOL, ATM, LSND and NEV, where NEV contains the experiments KARMEN [7], CDHS [8, Bugey [9] and $\mathrm{CHOOZ} /$ Palo Verde 10$]$, reporting no evidence for oscillations. Following Ref. [12] we con- 


\begin{tabular}{|c|rccc|c|}
\hline & SOL & ATM & LSND & NEV & $\chi_{\text {PG }}^{2}$ \\
\hline$(3+1)$ & 0.0 & 0.4 & 7.2 & 7.0 & 14.6 \\
$(2+2)$ & 14.8 & 6.7 & 2.2 & 9.7 & 32.4 \\
\hline
\end{tabular}

Table 1

Contributions of different data sets to $\chi_{\mathrm{PG}}^{2}$ in $(3+1)$ and $(2+2)$ schemes.

sider

$$
\begin{aligned}
\bar{\chi}^{2} & =\Delta \chi_{\mathrm{SOL}}^{2}\left(\theta_{\mathrm{SOL}}, \Delta m_{\mathrm{SOL}}^{2}, \eta_{s}\right) \\
& +\Delta \chi_{\mathrm{ATM}}^{2}\left(\theta_{\mathrm{ATM}}, \Delta m_{\mathrm{ATM}}^{2}, \eta_{s}, d_{\mu}\right) \\
& +\Delta \chi_{\mathrm{NEV}}^{2}\left(\theta_{\mathrm{LSND}}, \Delta m_{\mathrm{LSND}}^{2}, d_{\mu}, \eta_{e}\right) \\
& +\Delta \chi_{\mathrm{LSND}}^{2}\left(\theta_{\mathrm{LSND}}, \Delta m_{\mathrm{LSND}}^{2}\right)
\end{aligned}
$$

where $\Delta \chi_{X}^{2}=\chi_{X}^{2}-\left(\chi_{X}^{2}\right)_{\min }(X=\mathrm{SOL}$, ATM, NEV, LSND). In Tab. 1 we show the contributions of the 4 data sets to $\chi_{\mathrm{PG}}^{2} \equiv \bar{\chi}_{\mathrm{min}}^{2}$ for $(3+1)$ and $(2+2)$ oscillation schemes. As expected we observe that in $(3+1)$ schemes the main contribution comes from SBL data due to the tension between LSND and NEV data in these schemes. For $(2+2)$ oscillation schemes a large part of $\chi_{\mathrm{PG}}^{2}$ comes from solar and atmospheric data, however, also SBL data contributes significantly. This comes mainly from the tension between LSND and KARMEN [17], which does not depend on the mass scheme and, hence, also contributes in the case of $(2+2)$. Therefore, the values of $\chi_{\mathrm{PG}}^{2}$ in Tab. 1 for $(2+2)$ schemes are higher than the one given in Sec. 3, where the tension in SBL data is not included.

The parameter goodness of fit is now obtained by evaluating $\chi_{\mathrm{PG}}^{2}$ for 4 DOF 12 . This number of degrees of freedom corresponds to the 4 parameters $\eta_{s}, d_{\mu}, \theta_{\mathrm{LSND}}, \Delta m_{\mathrm{LSND}}^{2}$ describing the coupling of the different data sets (see Eq. (3)). The best GOF is obtained in the $(3+1)$ case. However, even in this best case the PG is only $0.56 \%$. The PG of $1.6 \times 10^{-6}$ for $(2+2)$ schemes shows that these mass schemes are essentially ruled out by the disagreement between the individual data sets.

Although we have seen that none of the fourneutrino mass schemes can provide a reasonable good fit to the global oscillation data including LSND, it might be interesting to consider the relative status of the three hypotheses $(3+1),(2+2)$ and the three-active neutrino scenario $(3+0)$. This can be done by comparing the $\chi^{2}$ values of the best fit point (which is in the $(3+1)$ scheme) with the one corresponding to $(2+2)$ and $(3+0)$. First we observe that $(2+2)$ schemes are strongly disfavoured with respect to $(3+1)$ with a $\Delta \chi^{2}=17.8$. The reason for the big change with respect to the value of $\Delta \chi^{2}=3.7$ found in Ref. [11] is the improved sensitivity of solar (SNO NC) and atmospheric (SK 1489-days) data to a sterile component. With this new data now $(3+1)$ schemes are clearly preferred over $(2+2)$ : for 4 DOF a $\Delta \chi^{2}=17.8$ implies that $(2+2)$ is ruled out at $99.87 \% \mathrm{CL}$ with respect to $(3+1)$. Further we find that $(2+2)$ is only slightly better than $(3+0)$, which is disfavoured with a $\Delta \chi^{2}=20.0$ with respect to $(3+1)$.

\section{CONCLUSIONS}

Using latest solar and atmospheric data, we re-analyze the four-neutrino description of current global neutrino oscillation data, including the LSND evidence for oscillations. The higher degree of rejection for non-active solar and atmospheric oscillation solutions implied by the SNO neutral current result as well as by the latest 1489-day SK atmospheric neutrino data allows us to rule out $(2+2)$ oscillation schemes. Using an improved goodness of fit method especially sensitive to the combination of data sets we obtain a GOF of only $1.6 \times 10^{-6}$ for $(2+2)$ schemes. Further, we find that also $(3+1)$ schemes are strongly disfavoured by the data. The disagreement between negative short-baseline experiments and LSND in $(3+1)$ schemes imply a GOF of $5.6 \times$ $10^{-3}$. This leads to the conclusion that all fourneutrino descriptions of the LSND anomaly, both in $(2+2)$ as well as $(3+1)$ realizations, are highly disfavoured. Our analysis brings the LSND hint to a more puzzling status, and the situation will become even more puzzling if LSND should be confirmed by the up-coming MiniBooNE experiment 18.

\section{REFERENCES}

1. S. Fukuda et al., Super-Kamiokande Coll., Phys. Lett. B 539 (2002) 179; B.T. Cleve- 
land et al., Astrophys. J. 496, 505 (1998); D.N. Abdurashitov et al., SAGE Coll., Phys. Rev. C60, 055801 (1999); astro-ph/0204245; W. Hampel et al., GALLEX Coll., Phys. Lett. B447, 127 (1999); C. Cattadori, GNO Coll., Nucl. Phys. B (Proc. Suppl.) 110 (2002) 311.

2. Q. R. Ahmad et al., SNO Coll., Phys. Rev. Lett. 89, 011301 (2002); Phys. Rev. Lett. 89, 011302 (2002).

3. M. Shiozawa, talk at Neutrino 2002, http://neutrino2002.ph.tum.de/; SuperKamiokande Coll., Y. Fukuda et al., Phys. Rev. Lett. 81 (1998) 1562.

4. MACRO Coll., M. Ambrosio et al., Phys. Lett. B 434 (1998) 451; A. Surdo, Talk given at TAUP 2001, 8-12 September 2001, Gran Sasso, Italy http://www. lngs.infn.it/.

5. A. Aguilar et al., LSND Coll., Phys. Rev. D 64 (2001) 112007.

6. J. T. Peltoniemi, D. Tommasini and J. W. F. Valle, Phys. Lett. B 298 (1993) 383; J. T. Peltoniemi and J. W. F. Valle, Nucl. Phys. B 406, 409 (1993); D.O. Caldwell and R.N. Mohapatra, Phys. Rev. D 48 (1993) 3259 .

7. B. Armbruster et al., KARMEN Coll., Phys. Rev. D 65 (2002) 112001.

8. F. Dydak et al., CDHS Coll., Phys. Lett. B 134 (1984) 281.

9. B. Achkar et al., Bugey Coll., Nucl. Phys. B 434 (1995) 503.

10. M. Apollonio et al., CHOOZ Coll., Phys. Lett. B 466 (1999) 415; F. Boehm et al., Palo Verde Coll., Phys. Rev. D 64 (2001) 112001.

11. M. Maltoni, T. Schwetz and J.W.F. Valle, Phys. Rev. D 65 (2002) 093004 hep$\mathrm{ph} / 0112103$.

12. M. Maltoni, T. Schwetz, M.A. Tórtola and J.W.F. Valle, hep-ph/0207157 (accepted for publication in Nucl. Phys. B).

13. M. Maltoni, T. Schwetz, M.A. Tórtola and J.W.F. Valle, hep-ph/0207227; Talk by M. Maltoni, these proceedings.

14. M.C. Gonzalez-Garcia, M. Maltoni and C. Pena-Garay, Phys. Rev. D 64 (2001) 093001 hep-ph/0105269].

15. S.M. Bilenky, C. Giunti and W. Grimus, in Proceedings of Neutrino '96, Helsinki, Fin- land, 13-19 June 1996, p. 174; Eur. Phys. J. C 1 (1998) 247; N. Okada and O. Yasuda, Int. J. Mod. Phys. A 12 (1997) 3669; V. Barger, et at., Phys. Rev. D 58 (1998) 093016; S.M. Bilenky, C. Giunti, W. Grimus and T. Schwetz, Phys. Rev. D 60 (1999) 073007; W. Grimus and T. Schwetz, Eur. Phys. J. C 20 (2001) 1 hep-ph/0102252.

16. M. Maltoni, T. Schwetz and J. W. F. Valle, Phys. Lett. B 518, 252 (2001) hep$\mathrm{ph} / 0107150$.

17. E.D. Church, K. Eitel, G.B. Mills and M. Steidl, Phys. Rev. D 66 (2002) 013001 hep-ex/0203023.

18. MiniBooNE Collaboration, R. Tayloe, Talk at Neutrino 2002, Munich, http://neutrino2002.ph.tum.de/. 